\title{
Soybean Oil-Based Lipid Emulsion Increases Intestinal Permeability of Lipopolysaccharide in Caco-2 Cells by Downregulation of P-Glycoprotein via ERK-FOXO 3a Pathway
}

\author{
Jun-Kai Yanª Jie Zhua,b Bei-Lin Gu ${ }^{a, b}$ Wei-Hui Yana,b Yong-Tao Xiao ${ }^{a, b}$ \\ Ke-Jun Zhou ${ }^{a, b}$ Jie Wen ${ }^{a, b}$ Yang Wanga,b Wei Cai ${ }^{a, b}$ \\ aXin Hua Hospital affiliated to Shanghai Jiao Tong University School of Medicine, 'bhanghai Key \\ Laboratory of Pediatric Gastroenterology and Nutrition, Shanghai Institute for Pediatric Research, \\ Shanghai, China
}

\section{Key Words}

Parenteral nutrition $\cdot$ Soybean oil-based lipid emulsion $\cdot$ P-glycoprotein $\cdot$ Lipopolysaccharide - Caco-2

\begin{abstract}
Background and Aims: Elevated intestinal permeability of lipopolysaccharide (LPS) is a major complication for patients with parenteral nutrition (PN), but the pathogenesis is poorly understood. Intestinal P-glycoprotein (P-gp) is one of the efflux transporters that contribute to restricting the permeability of lipopolysaccharide via transcellular route. P-gp expression may be regulated by PN ingredients, and thus this study sought to investigate the effect of PN on the expression of P-gp and to elucidate the underlying mechanism in vitro. Methods: Caco-2 cells were treated with PN ingredients. Changes in P-gp expression and function were determined and the role of ERK-FOXO 3a pathway was studied. Transport studies of FITC-lipopolysaccharide (FITC-LPS) across Caco-2 cell monolayers were also performed. Results: Among PN ingredients, soybean oil-based lipid emulsion (SOLE) exhibited significant inhibitory effect on P-gp expression and function. This regulation was mediated via activation of ERK pathway with subsequent nuclear exclusion of FOXO 3a. Importantly, P-gp participated in antagonizing the permeation of FITC-LPS (apical to basolateral) across Caco-2 cell monolayers. SOLE significantly increased the permeability of FITC-LPS (apical to basolateral), which was associated with impaired P-gp function. Conclusions: The expression and function of intestinal P-gp is suppressed by SOLE in vitro.
\end{abstract}

\section{Introduction}

(C) 2016 The Author(s)

Published by S. Karger AG, Basel

Total parenteral nutrition (TPN) is vital for the nutritional support of patients with loss of anatomical or functional integrity of the gastrointestinal tract. However, it also leads to 
elevated intestinal permeability of lipopolysaccharide (LPS) which may cross the intestinal barrier and enter the systemic circulation, in turn leading to gut-derived sepsis or parenteral nutrition associated liver disease (PNALD) [1, 2]. Alternatively, it may be trapped in the intestinal wall or mesenteric lymph nodes and stimulate the production of various cytokines by the lymphatic system, contributing to the systemic inflammatory response syndrome (SIRS) [3-5]. Currently, the exact mechanism counting for elevated intestinal permeability of LPS under TPN has not been fully elucidated. Although it is generally suggested that impaired intestinal barrier function is the major cause of paracellular permeability of LPS to underlying tissues $[6,7]$, it is not clear whether the permeability of LPS is facilitated by TPN via transcellular route.

P-glycoprotein (P-gp), a $170 \mathrm{kDa}$ phosphorylated and glycosylated plasma membrane protein which belongs to the ATP binding cassette super family, is found richly expressed on the apical surfaces of superficial columnar epithelial cells of the colon and small bowel. Given its apical distribution on the enterocytes and efflux pump activity, P-gp is exquisitely positioned to limit the absorption of harmful substances from the gut [8]. Conceivably, while intestinal barrier participates in restricting the permeability of LPS via paracellular route, intestinal P-gp may contribute to preventing its permeation via transcellular route. Thus, whether the expression of intestinal P-gp is altered by TPN and what is the clinical significance beyond this alteration need to be addressed.

Obviously, artificial PN ingredients, which include lipid emulsion, amino acids, carbohydrates, electrolytes, vitamins, and trace elements, are able to affect intestinal homeostasis in a variety of aspects. Recently, in an in vivo study using rodent models of TPN, striking differences were noted when varying lipid emulsions were used in terms of small bowel mucosa-derived cytokines, animal survival rate, epithelial cell proliferation and apoptosis rates, intestinal barrier function and mucosal fatty acids composition [9]. These findings aroused our great interest in the role of lipid emulsions in P-gp regulation. At present, commercially available products of lipid emulsions include: soybean oil-based Lipofundin, olive oil-supplemented Clinoleic, fish oil-derived Omegaven, and a combination of soybean oil, olive oil and fish oil (SMOF). Though most of these have been widely studied in terms of system effects, little is known regarding their impacts on enterocytes.

Taken together, we hypothesize that intestinal P-gp may be regulated by SOLE under TPN, which consequently facilitate the permeability of LPS via transcellular route. To test this, human intestine-derived Caco-2 cells were used, which has been approved suitable for studying PN in vitro previously elsewhere [10]. In addition to intestinal barrier function, our findings may provide a new perspective on the elevated permeability of LPS during TPN which involves lipid emulsion and P-gp function.

\section{Material and Methods}

\section{Chemicals}

Cell culture reagents were purchased from Life Technologies (Shanghai, China). Rabbit anti p-ERK, ERK, p-FOXO 3a, FOXO 3a, GAPDH, and mouse anti P-gp were purchased from Abcam (Shanghai, China). Rabbit anti ZO-1 and horseradish peroxidase conjugated anti-mouse and anti-rabbit IgG were purchased from CST (Shanghai, China). PN solution was obtained from clinical nutrition department, Xin Hua Hospital. Composition of PN solution is presented in Table 1. Regarding lipid emulsion, we chose soybean oil-based lipid emulsion (SOLE) as representative because it is used more extensively in clinical practice. SOLE was derived from commercially available Lipofundin (20\% LCT/MCT, Baxter Healthcare, China). All other chemicals were purchased from Sigma (Shanghai, China).

\section{Cell culture}

Caco-2 cells (American Type Culture Collection, USA) were maintained under standard cell culture conditions as described previously [11]. For transport studies, cell monolayer integrity on Transwell inserts (12 mm diameter, $0.4 \mu \mathrm{m}$ pore size; Corning Costar Co., USA) was tested by measuring transepithelial 


\section{Cellular Physiology Cell Physiol Biochem 2016;39:1581-1594

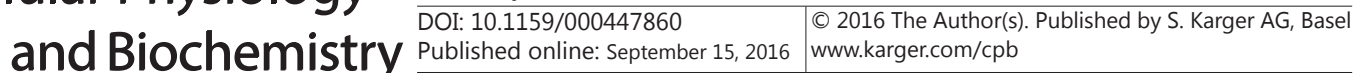 \\ Yan et al:: Soybean Oil Downregulates P-gp Expression}

Table 1. Composition of PN solution

\begin{tabular}{llll}
\hline Components & Volume $(\mathrm{mL})$ & Kcal & \% of Calories \\
\hline $50 \%$ glucose & 40 & 80.0 & 64.44 \\
$8.5 \%$ amino acids & 30 & 10.20 & 7.83 \\
$20 \%$ SOLE (lipofundin) & 20 & 40.0 & 30.72 \\
$10 \%$ sodium chloride & 3 & & \\
$10 \%$ potassium chloride & 2 & & \\
$10 \%$ calcium gluconate & 2 & & \\
Water-soluble vitamins (Soluvit) & 0.2 & & \\
Fat-soluble vitamins (Vitalipid) & 0.2 & & \\
Multi-trace Elements Injection & 0.2 & & \\
Sodium Glycerophosphate Injection & 0.2 & 130.2 & 100 \\
Total & 97.8 & & \\
\hline
\end{tabular}

electrical resistance (TEER). Only monolayers displaying TEER values above $300 \Omega \cdot \mathrm{cm}^{2}$ were considered not leaky and suitable for subsequent studies.

\section{Cell treatment}

Caco- 2 cells were respectively exposed to $1 \% \mathrm{PN}$ (vol/vol dissolved in culture medium), $0.4 \%$ glucose, $0.3 \%$ amino acids, $0.2 \%$ SOLE, $1 \%$ PN without glucose, $1 \%$ PN without amino acids, and $1 \%$ PN without SOLE to screen out the effective ingredient that may elicit the regulatory effect on P-gp. Regarding these ingredients one aspect requires comment. According to Table 1, glucose, SOLE and amino acids account for $40 \%, 20 \%$ and $30 \%$ of whole solution, respectively. Therefore, if $1 \%$ PN was used, the corresponding doses of glucose, SOLE and amino acid should be $0.4 \%, 0.2 \%$ and $0.3 \%$, respectively. As SOLE was approved effective on P-gp regulation, cells were exposed to various concentrations of SOLE $(0.05 \%, 0.1 \%$, and $0.2 \%$ vol/vol dissolved in culture medium) for 24-48 hours to further investigate its effect on P-gp expression and function in vitro. When specific inhibitors were used, ERK inhibitor PD98059 (25 $\mu \mathrm{M})$ was added to the medium $1 \mathrm{~h}$ prior to the treatment with SOLE. As the stock solution of inhibitors was dissolved in DMSO, an equal volume of DMSO (final concentration $<0.1 \%$ ) was added to the control cells.

Intracellular rhodamine 123 accumulation assay and transport studies

P-gp function was evaluated by measuring intracellular accumulation of rhodamine 123 (a specific substrate for P-gp) as described previously [12]. Briefly, cells were incubated with $10 \mu \mathrm{M}$ rhodamine 123 in culture medium in the dark at $37^{\circ} \mathrm{C}$ for 60 minutes. After incubation, cells were washed five times with ice-cold PBS and then lysed with 1\% Triton X-100. The amount of rhodamine 123 was determined using a fluorescence spectrophotometer (SpectraMax, CA, USA) with an excitation wavelength of $488 \mathrm{~nm}$ and emission wavelength of $530 \mathrm{~nm}$, and normalized to protein content. For transport studies, cells were exposed to SOLE $(0.2 \% \mathrm{vol} / \mathrm{vol}$ added to basolateral medium). The integrity of monolayers was checked by TEER as described above. Rhodamine $123(5 \mu \mathrm{M})$ was added into either the basolateral side for the basolateral to apical (BL>AP) transport study or apical side for the apical to basolateral (AP>BL) transport study. At designated times, $50 \mu \mathrm{l}$ aliquots were sampled, with replacement, every 30 min from the receiver chamber. Verapamill $(100 \mu \mathrm{M})$ was used for specific inhibition of P-gp function.

\section{Calculations}

The apparent permeability coefficients (Papp coefficients) were calculated for the directional flux studies according to the following equation:

Papp $=(\mathrm{dQ} / \mathrm{dt}) /\left(\mathrm{A} \times \mathrm{C}_{0}\right)$

where the $\mathrm{dQ} / \mathrm{dt}(\mu \mathrm{mol} / \mathrm{min})$ is the drug permeation rate, $\mathrm{A}$ is the cross sectional area $\left(1.1 \mathrm{~cm}^{2}\right)$, and $\mathrm{C}_{0}(\mu \mathrm{M})$ is the initial concentration in the donor compartment at $\mathrm{t}=0 \mathrm{~min}$. The net efflux (Papp ratio) is expressed as the quotient of Papp (BL>AP) to Papp (AP>BL)

\section{Intracellular FITC-LPS accumulation studies and transport studies}

Transport studies for FITC-LPS were performed as described previously [13]. Briefly, FITC-LPS (50 $\mu \mathrm{g} / \mathrm{ml}$ ) was added into the apical chamber for apical to basolateral (AP>BL) transport study. At designated times, $50 \mu \mathrm{l}$ aliquots were sampled, with replacement, every $30 \mathrm{~min}$ from basolateral chamber. After the experiments, monolayers were rinsed five times with ice-cold PBS and lysed with 1\% Triton X-100. The 


\section{Cellular Physiology Cell Physiol Biochem 2016;39:1581-1594 \begin{tabular}{l|l|l} 
and Biochemistry Published online: September 15, 2016 & $\begin{array}{l}\text { (c) } 2016 \text { The Author(s). Published by S. Karger AG, Basel } \\
\text { www.karger.com/cpb }\end{array}$
\end{tabular} \\ Yan et al.: Soybean Oil Downregulates P-gp Expression}

Table 2. Primers used for quantitative real-time PCR analysis

\begin{tabular}{lll}
\hline Gene & Primer sequence $\left(5^{\prime}-3^{\prime}\right)$ & Amplicon size \\
\hline P-gp & Forward: GTTCTTTCAGTCAATGGCAAC & 211 \\
& $\begin{array}{l}\text { Reverse: TCTAATTGCTGCCAAGACCTC } \\
\text { GAPDH }\end{array}$ & $\begin{array}{l}\text { Forward: TATTGTTGCCATCAATGACCC } \\
\text { Reverse: ACTCCACGACGTACTCAGC }\end{array}$ \\
& 206 \\
\hline
\end{tabular}

amount of FITC-LPS was determined using a fluorescence spectrophotometer and normalized to protein content. Verapamill $(100 \mu \mathrm{M})$ was used for specific inhibition of P-gp function.

mRNA stability assay

Caco- 2 cells were treated with Actinomycin D (Act D, at $5 \mu \mathrm{g} / \mathrm{ml}$ ) for the indicated periods as described previously [14]. The amount of mRNA at $0 \mathrm{~h}$ time point was set as $100 \%$ and thereafter, the percentage of normalized P-gp mRNA levels versus time was plotted to calculate the half-life of P-gp transcripts.

\section{Quantitative real-time PCR}

Total RNA was extracted from Caco-2 cells using Trizol (life technology, USA), and cDNA was synthesized with an RNA isolation plus kit (Takara Shuzo, Japan) according to the manufacturer's protocol. The amplification program consisted of activation at $95{ }^{\circ} \mathrm{C}$ for $5 \mathrm{~min}$, followed by 35 amplification cycles, each consisting of $95{ }^{\circ} \mathrm{C}$ for $15 \mathrm{~s}$ then $60^{\circ} \mathrm{C}$ for $1 \mathrm{~min}$. The primers used are shown in Table 2. Data was analyzed using PIK096 software (Thermo, Germany).

\section{Western blot analysis}

Cells were collected and washed twice with PBS. For total protein extraction, the harvested cells were lysed on ice for $30 \mathrm{~min}$ in $100 \mu \mathrm{l}$ of RIPA buffer and centrifuged at $12000 \mathrm{~g}$ for $10 \mathrm{~min}$. The supernatants were collected, and protein concentrations were determined using a BCA protein assay kit (Thermo, USA). Aliquots of the lysates (30 $\mu \mathrm{g}$ of protein) were loaded. Immunodetection was performed with enhanced chemiluminescence detection system (GE Healthcare, USA).

Preparation of nuclear and cytoplasmic extracts

Nuclear and cytolasmic extracts were prepared with a commercial kit according to the manufacturer's instructions (Thermo, USA). Protease inhibitor cocktail (Thermo, USA) and phosphatase inhibitor cocktail (Thermo, USA) were added to each buffer just prior to use. Lamin B and GAPDH served as loading control for nuclear fractions and cytoplasmic fractions, respectively.

\section{Immunofluorescence staining}

Cells were fixed in $4 \%$ formaldehyde at room temperature for $15 \mathrm{~min}$, and permeabilized with $0.2 \%$ Triton-X100 in PBS for 15 min. Cell monolayers were blocked in 5\% BSA and incubated with antibodies against ZO-1, P-gp, and p-FOXO 3 a at $4{ }^{\circ} \mathrm{C}$ overnight, followed by incubation with Alexa Fluor 488 or Alexa Fluor 594 conjugated secondary antibodies for $1 \mathrm{~h}$ at room temperature. Images were visualized using Leica DMI6000B fluorescence microscopy with LAS AF LITE image processing software (Leica, Germany).

\section{Statistical analysis}

Data in the Figures and text are expressed as means \pm standard deviation (S.D.) of at least three experiments each performed in triplicate. Statistical analysis employed Student's $t$ test for comparison of two means, and a one-way ANOVA for comparison of multiple groups. A significant difference between means was considered to be present when $\mathrm{P}<0.05$.

\section{Results}

Effects of PN solution and its main ingredients on the expression and function of P-gp

As shown in Fig. 1, treatment with PN solution in vitro significantly reduced the mRNA expression of P-gp (approximately $40 \%$ compared to control, $\mathrm{p}<0.05$ ). Consistently, rhodamine 123 accumulation was also significantly increased (approximately 200\% compared to KARGER 
Fig. 1. Effects of the PN ingredients on the expression and function of P-gp in vitro. (A) Effects of the $\mathrm{PN}$ ingredients on the mRNA expression of P-gp. Forty eight hours post treatment, mRNA expression of P-gp was determined by real-time PCR. Among PN ingredients, only SOLE significantly down-regulated P-gp expression. Data were normalized to GAPDH mRNA levels, and values are shown as the means \pm SD of data from triplicate experiments. *, p $<0.05$ compared with control. (B) Effects of the PN ingredients on the function of p-gp. Forty eight hours post treatment, cells were incubated with rhodamine 123 (10 $\mu \mathrm{M}$ ) for 1 hour. After incubation, intracellular concentrations of rhodamine 123 were determined and normalized to protein content. Consistent with P-gp expression, P-gp function was significantly suppressed by SOLE treatment. Values are shown as the means \pm SD of data from triplicate experiments. *, $p<0.05$ compared with control. Note that doses of each main ingredient were referenced to Table 1 as described in material and methods.

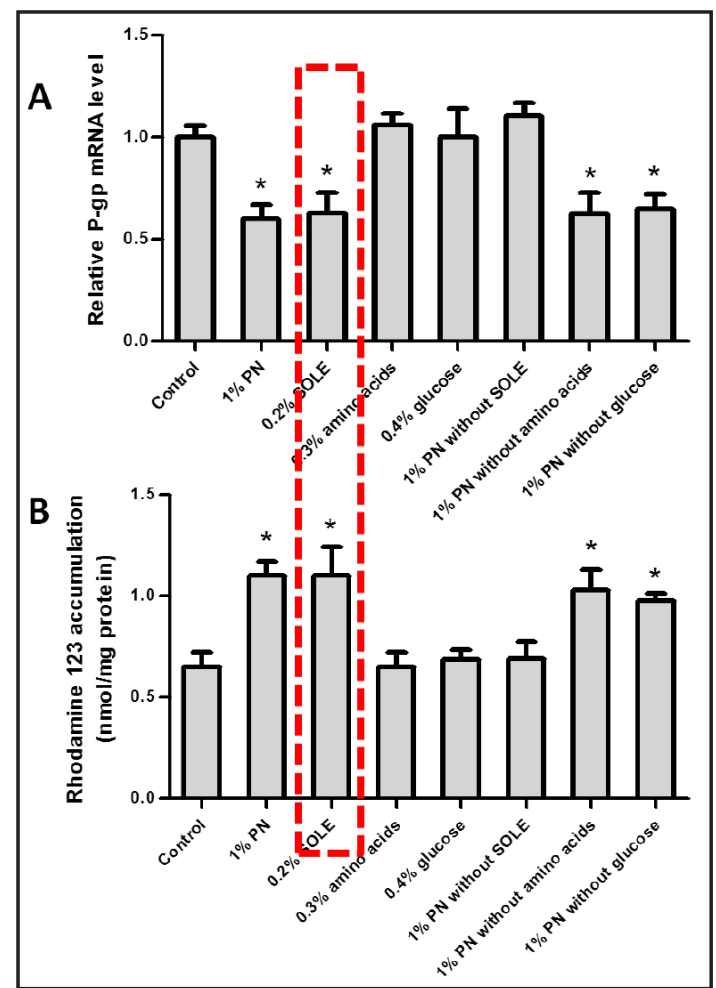

control, $\mathrm{p}<0.05$ ) in cells treated with PN solution. Among these main ingredients, only $0.2 \%$ SOLE elicited this regulatory effect on P-gp expression and function while no significant differences were observed upon treatment with glucose or amino acids.

SOLE down-regulates the expression of P-gp in a dose- and time-dependent manner

As shown in Fig. 2A, mRNA expression of P-gp was decreased on SOLE treatment in a dose-dependent manner. Compared with control, it was significantly decreased to $87.9 \%, 68.4 \%(p<0.05)$ and $52.7 \%(p<0.05)$ after treated with SOLE at $0.05,0.1$ and $0.2 \%$, respectively (Fig. 2A-a). Western blot analysis further substantially mirrored the results of real-time PCR (Fig. 2A-b). Since treatment with $0.2 \%$ SOLE elicited the most significant impact on P-gp expression, this concentration was further used in the time course study. As can be seen, P-gp expression was decreased on SOLE treatment in a time-dependent manner on both mRNA levels (Fig 2B-a) and protein levels (Fig. 2B-b). Consistently, compared with control $(0.62 \mathrm{nmol} / \mathrm{mg}$ protein), intracellular accumulation of rhodamine 123was significantly increased to $0.67,0.81$, and $0.95 \mathrm{nmol} / \mathrm{mg}$ protein after treated with SOLE at $0.05,0.1$ and $0.2 \%$, respectively (Fig. 2C). Taken together, these results suggested that SOLE down-regulated the expression of P-gp in a dose- and time-dependent manner

\section{SOLE increases the permeability of rhodamine 123 across Caco-2 cell monolayers}

The time course of rhodamine 123 transport was examined after addition of $5 \mu \mathrm{M}$ rhodamine 123 to either apical compartment or basolateral compartment of the Caco-2 monolayers. As can be seen, transport in the efflux direction (BL>AP) over the period of $2 \mathrm{~h}$ was significantly decreased by SOLE treatment (Fig. 3a). Specifically, the cumulative amount of Rhodamine 123 transport in the efflux direction was $145 \pm 15,85 \pm 20$ and $51 \pm 9$ $\mathrm{pmol} /$ monolayer for control cells, cells treated with SOLE and cells treated with verapamil, respectively. Transport in the uptake direction ( $\mathrm{AP}>\mathrm{BL}$ ) over the period of $2 \mathrm{~h}$ was significantly increased by SOLE treatment (Fig. 3b). Specifically, the cumulative amount of Rhodamine 123 transport in the uptake direction was $25 \pm 5,47 \pm 4$ and $35 \pm 8 \mathrm{pmol} / \mathrm{monolayer}$ for control cells, cells treated with SOLE and cells treated with verapamil, respectively. The Papp values were calculated and listed in Table 3. As indicated, Papp in the BL $>$ AP direction was decreased 


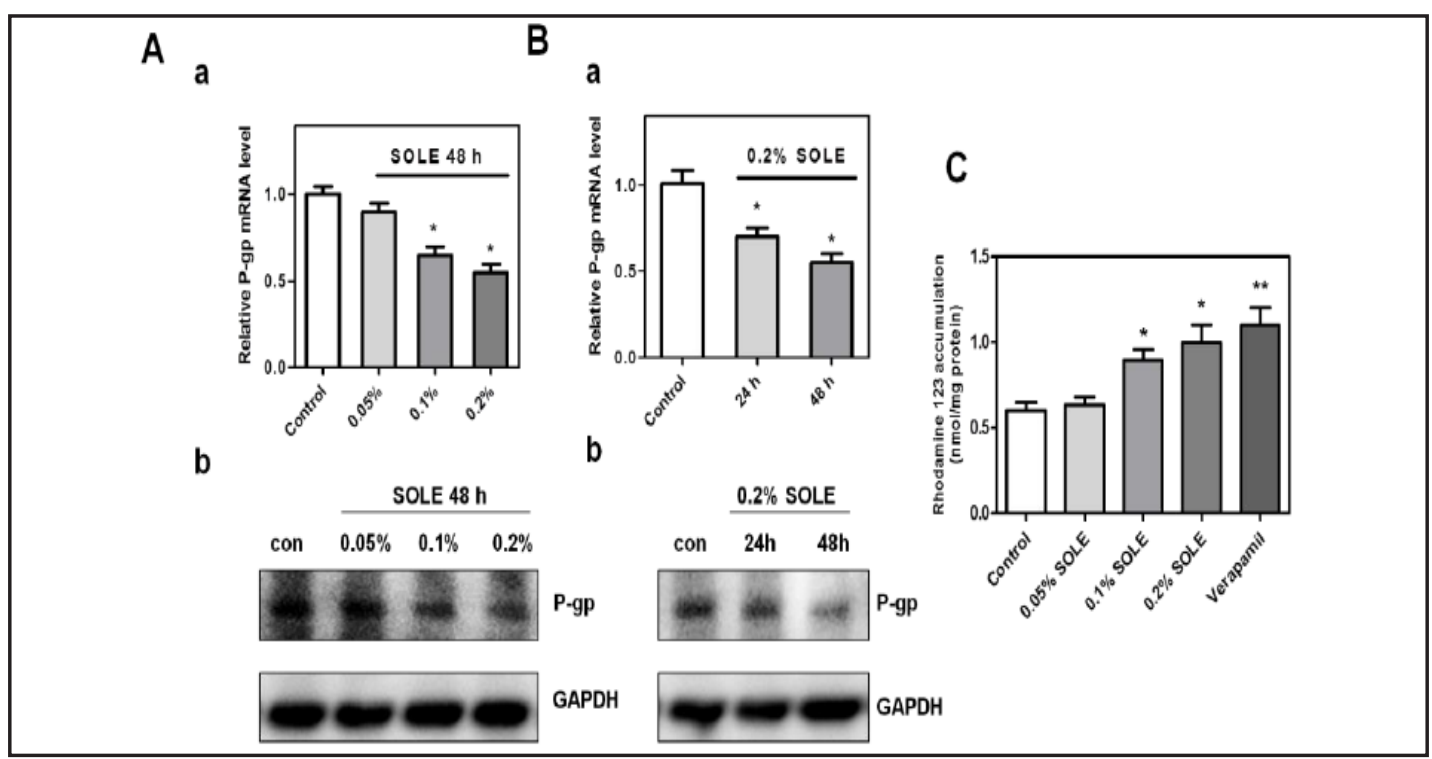

Fig. 2. SOLE down-regulated the expression of p-gp dose-dependently and time-dependently. (A) SOLE down-regulated the protein expression of p-gp in a dependent manner on both mRNA levels (a) and protein levels (b). Data were normalized to GAPDH mRNA levels, and values are shown as the means \pm SD of data from triplicate experiments. ${ }^{*}, \mathrm{p}<0.01$ compared with control. (B) SOLE down-regulated the mRNA expression of p-gp in a time-dependent manner on both mRNA levels (a) and protein levels (b). Data were normalized to GAPDH mRNA levels, and values are shown as the means \pm SD of data from triplicate experiments. *, p<0.01 compared with control. (C) SOLE increased the intracellular accumulation of rhodamine 123 in Caco-2 cells. Forty eight hours post treatment, cells were incubated with rhodamine $123(10 \mu \mathrm{M})$ for 1 hour. After incubation, intracellular concentrations of rhodamine 123 were determined and normalized to protein content. A specific inhibitor for P-gp (verapamil, $100 \mu \mathrm{M}$ ) served as positive control. Values are shown as the means \pm SD of data from triplicate experiments. ${ }^{*}, \mathrm{p}<0.05,{ }^{* *}, \mathrm{p}<0.01$ compared with control.

Table 3. Effect of SOLE on Papp of Rhodamine123 (5 $\mu \mathrm{M})$ across Caco-2 Monolayers. Results are reported as mean $\pm \mathrm{SD}, \mathrm{n}=3 .^{*}, \mathrm{p}<0.05$ compared to control

\begin{tabular}{llll} 
& \multicolumn{2}{l}{ Papp $\left(\times 10^{-6} \mathrm{~cm} / \mathrm{s}\right)$} & Net efflux ratio \\
& $\mathrm{BL}>\mathrm{AP}$ & $\mathrm{AP}>\mathrm{BL}$ & $\mathrm{Papp}(\mathrm{BL}>\mathrm{AP}) / \mathrm{Papp}(\mathrm{AP}>\mathrm{BL})$ \\
\hline Control & $3.79 \pm 0.39$ & $0.63 \pm 0.15$ & 6.01 \\
$0.2 \%$ SOLE & $2.15 \pm 0.28^{*}$ & $1.09 \pm 0.22^{*}$ & 1.97 \\
Verapamil & $1.31 \pm 0.33^{*}$ & $0.88 \pm 0.17^{*}$ & 1.48 \\
\hline
\end{tabular}

Table 4. P-gp function and mRNA expression in cells treated with SOLE or SOLE+PD98059. Results are reported as mean \pm SD, $n=3$. a, $p<0.05$ compared to control; b, p<0.05 compared to SOLE

\begin{tabular}{lccc}
\hline & Control & SOLE & SOLE+PD98059 \\
\hline $\begin{array}{l}\text { P-gp mRNA level } \\
\text { (Normalized) }\end{array}$ & $1.00 \pm 0.05$ & $0.55 \pm 0.09^{\mathrm{a}}$ & $0.75 \pm 0.07^{\mathrm{b}}$ \\
$\begin{array}{l}\text { Rhodamine 123 accumulation } \\
\text { (nmol/mg protein) }\end{array}$ & $0.52 \pm 0.07$ & $1.15 \pm 0.05^{\mathrm{a}}$ & $0.79 \pm 0.11^{\mathrm{b}}$ \\
\hline
\end{tabular}

by $44 \%$ and increased by $73 \%$ in the AP>BL direction, leading to $67 \%$ reduction in the net efflux ratio on SOLE treatment. Taken together, these results suggested that intestinal P-gp function was markedly impaired by SOLE treatment in vitro.

\section{Down-regulation of P-gp does not involve mRNA degradation}

As indicated in Fig. 2, down-regulation of P-gp by SOLE was already evident on mRNA levels. As reduced mRNA abundance could be resulted from either a decrease in mRNA transcription or an increase in mRNA decay, to test this, mRNA stability assay was performed. As shown in Fig. 4, the half-life of P-gp in control cells and that in SOLE-treated cells was 
Fig. 3. SOLE suppressed the efflux function of P-gp in Caco2 monolayers. Caco- 2 cells were exposed to SOLE $(0.2 \%$, added to the basolateral medium) for 48 hours. Rhodamine $123(5 \mu \mathrm{M})$ was either added to the basolateral side for $\mathrm{BL}>\mathrm{AP}$ transport study (a) or apical side for AP>BL transport study (b). The amounts of rhodamine

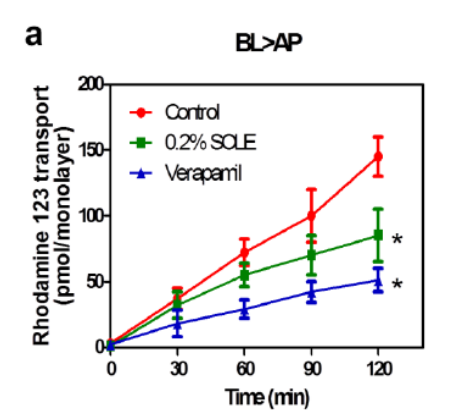

b

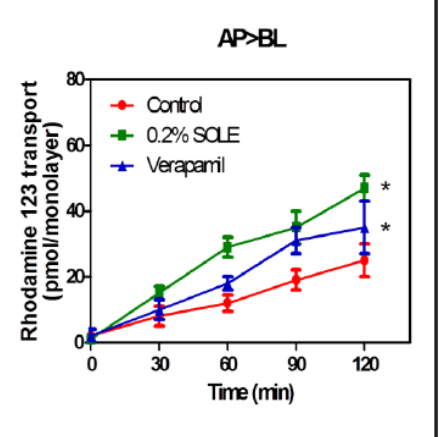

123 in the receiver compartment were determined at 30, 60, 90, 120 min after incubation. Each point represents the means $\pm \mathrm{SD}$ ( $\mathrm{n}=3$ for each group). ${ }^{*}, \mathrm{p}<0.05$ compared with control. Verapamil served as positive control. The results showed that the transport of rhodamine 123 was significantly decreased in the BL $>A P$ direction (a) but increased in the AP>BL direction (b) in cells treated with SOLE.

Fig. 4. Stability of p-gp mRNA was not affected by SOLE treatment. Caco-2 cells were exposed to $0.2 \%$ SOLE for 24 hours, and then further treated with the RNA polymerase inhibitor actinomycin D for the indicated periods. Total RNAs were prepared, and P-gp mRNA level was analyzed by real-time PCR. The results showed that the half-life of P-gp mRNA was not affected by SOLE treatment.

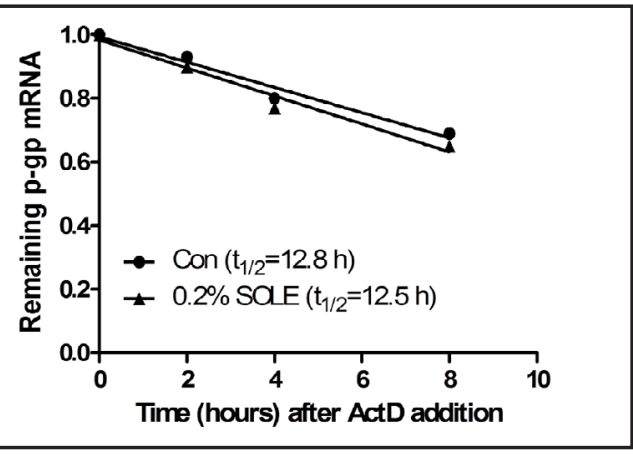

Fig. 5. SOLE down-regulated P-gp expression via ERK pathway. (A) Treatment with SOLE activated ERK pathway. Caco-2 cells were exposed to $0.2 \%$ SOLE for various time $(0,5,15,60$, and $120 \mathrm{~min})$, followed by determining the phosphorylation level and total amount of ERK. (B) Blocking the ERK pathway with PD98059. Specific inhibitor PD98059 $(25 \mu \mathrm{M})$ was added to the medium $1 \mathrm{~h}$ prior to the addition of SOLE to block the activation of ERK pathway. (C) Blocking the activation of ERK pathway partly attenuated the inhibitory impact of SOLE on P-gp expression.

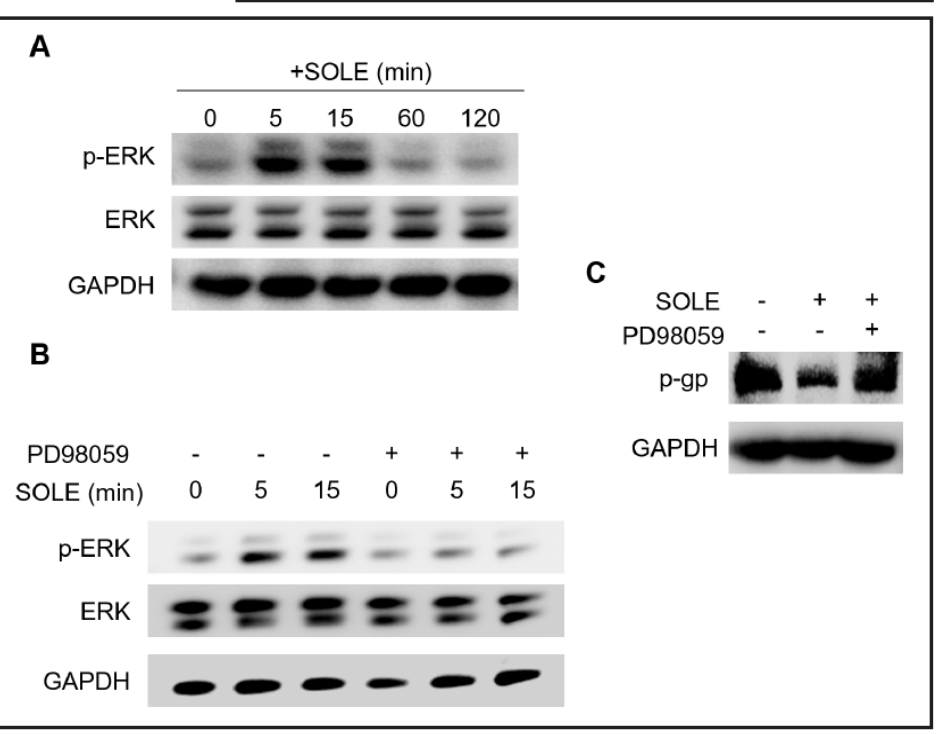

$12.8 \mathrm{~h}$ and $12.5 \mathrm{~h}$, respectively. No significant differences in mRNA stability were observed between two groups, suggesting that mRNA degradation was not involved in the downregulation of P-gp by SOLE treatment.

SOLE down-regulates P-gp expression via ERK pathway

In an attempt to further understand the mechanisms, we investigated whether ERK pathway contributed to the regulation of P-gp by SOLE treatment. First of all, the activation of ERK pathway in response to SOLE was confirmed. As shown, though no changes of total amount of ERK were observed within the period of time $(0,5,15,60$, and 120 
Fig. 6. SOLE induced nuclear exclusion of FOXO 3a via ERK pathway. (A) SOLE induced nuclear exclusion of FOXO 3a. Caco-2 cells were incubated with SOLE for the indicated periods. Cells were then harvested and the total amount of protein as well as the nuclear and cytoplasmic fractions were collected and analyzed by western blot with the indicated antibodies. As indicated, SOLE activated ERK pathway without changing the subsequent phosphorylation levels and total amount of FOXO 3a. However, p-FOXO 3a levels were reduced in the nucleus but increased in the cytoplasm with SOLE treatment, suggesting that p-FOXO $3 a$ was translocated from the nucleus to the cytoplasm (a). Analyzed by band intensity, the results showed that subcellular distribution of p-FOXO 3a (nuclear/cytoplasmic fraction) was significantly changed within the indicated period of time (b). (B) Blocking of ERK pathway

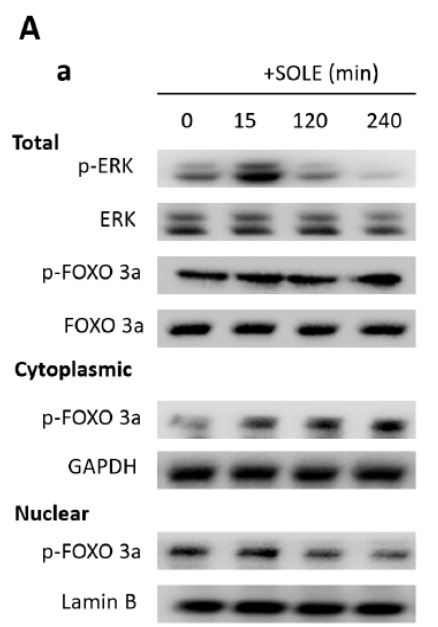

C

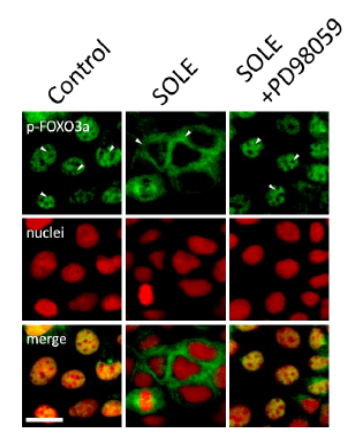

b

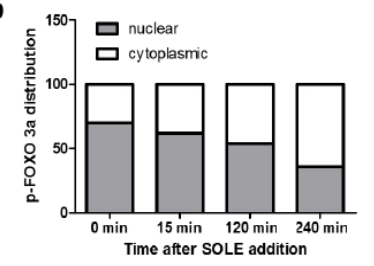

B

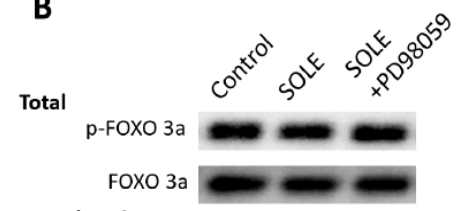

Cytoplasmic

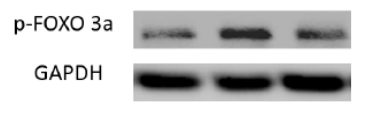

Nuclear

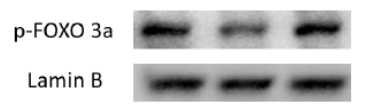

D

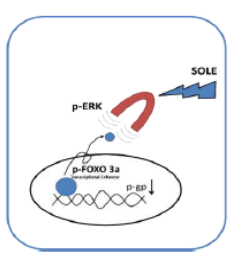
with specific inhibitor reversed the nuclear exclusion of FOXO 3a. Specific inhibitor PD98059 (25 $\mu \mathrm{M})$ was added to the medium $1 \mathrm{~h}$ prior to the addition of SOLE to block the activation of ERK pathway. Cells were harvested after incubation with SOLE for the indicated periods and the total amount of protein as well as the nuclear and cytoplasmic fractions were collected. As shown, compared to SOLE alone, cytoplasmic p-FOXO 3a was significantly decreased while nuclear p-FOXO 3a was increased in SOLE with PD98059. (C) Representative immunofluoresence staining for p-FOXO3a. Note a predominant nuclear localization of p-FOXO 3a (green) in control cells and a predominant cytoplasmic localization of p-FOXO 3 a in the cells treated with SOLE, as indicated by the white arrows. Blocking of ERK pathway with PD98059 relieved the SOLE-induced cytoplasmic localization of p-FOXO 3a.Scale bar $=25 \mu \mathrm{m}$. Three experiments were performed that showed similar results. (D) Schematic representation showing that SOLE activates ERK pathway, leading to the nuclear exclusion of FOXO $3 \mathrm{a}$ and subsequent down-regulation of P-gp transcription.

min), phosphorylated levels of ERK (p-ERK) were evident already at 5-15 min after SOLE stimulation and decreased until $60 \mathrm{~min}$ (Fig. 5A). When specific inhibitor for ERK pathway PD98059 was used (Fig. 5B), SOLE-induced decline in P-gp expression was partly attenuated on protein levels (Fig. 5C). Consistently, P-gp function as well as its mRNA expression was partly attenuated by blocking of ERK pathway (Table 4). Taken together, these results suggested that ERK pathway may play a pivotal role in mediating the regulation of intestinal P-gp by SOLE treatment in vitro.

\section{SOLE activates ERK pathway and induces the nuclear exclusion of FOXO $3 a$}

As one of the downstream molecules of ERK pathway, FOXO 3a was examined because: 1) it functions as an enhancer of P-gp transcription [15]; 2) once phosphorlyated by ERK, it could translocate from nucleus to cytoplasm and lose its transcriptional activity [16]. Thus, we hypothesized that SOLE may induce activation of ERK pathway, leading to the nuclear exclusion of FOXO $3 \mathrm{a}$ and the consequent reduction in P-gp transcription. As shown in Fig. KARGER 
A

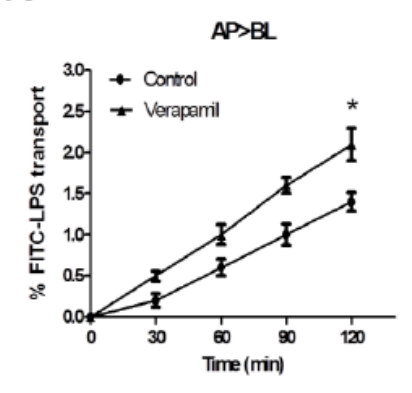

B

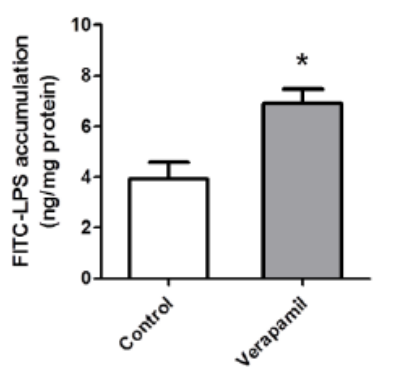

C

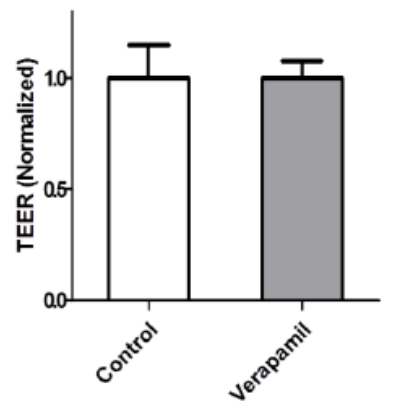

Fig. 7. FITC-LPS permeability was increased in Caco-2 cells with impaired function of P-gp. (A) The time course of FITC-LPS permeation across Caco-2 cell monolayers. FITC-LPS (50 $\mu \mathrm{g} / \mathrm{ml})$ was added to apical side in the presence or absence of the P-gp specific inhibitor verapamil $(100 \mu \mathrm{M})$. The amounts of FITC-LPS in the receiver compartment were determined at 30, 60, 90, 120 min after incubation. Each point represents the means $\pm S D$ ( $n=3$ for each group). As shown, permeability of FITC-LPS was significantly increased when the cells were incubated with verapamil (a). *, p<0.05 compared to control. (B) Intracellular accumulation of FITC-LPS in cells with treated with verapamil. Following the 120 min-transport, membranes were rinsed with PBS and the cells were lysed with RIPA. Intracellular accumulation of FITC-LPS was determined and normalized to protein content. As shown, FITC-LPS accumulation was significantly increased in the cells treated with verapamil. Values are shown as the means \pm SD ( $n=3$ for each group). *, $\mathrm{p}<0.05$ compared to control. (C) Integrity of monolayers was unchanged upon verapamil treatment. Cell integrity was evaluated by TEER following the 120 min-transport and no significant differences were observed.

$6 \mathrm{~A}$, neither the phosphorylation level nor the total amount of FOXO 3a was changed with the activation of ERK pathway in the whole cell lysate. However, phosphorylated levels of FOXO 3a (p-FOXO 3a) were reduced in the nucleus but increased in the cytoplasm with SOLE treatment over the period of time, suggesting that p-FOXO 3a translocated from the nucleus to the cytoplasm in response to SOLE treament. By measuring band intensity, the results revealed that subcellular distribution (nuclear/cytoplamic) of p-FOXO 3a shifted from 2:1 to 1:2 upon SOLE treatment. Importantly, blocking of ERK pathway abolished the SOLEinduced redistribution of p-FOXO 3a, though p-FOXO 3a and FOXO 3a in whole cell lysate were still unchanged (Fig. 6B) Representative images indicating the translocation of p-FOXO 3a are shown in Fig. 6C and a schematic representation is shown in Fig. 6D. These results suggested that SOLE induced nuclear exclusion of p-FOXO 3a, which was dependent on the activation of ERK pathway.

FITC-LPS permeability is increased in Caco-2 cell monolayers with impaired function of $P-g p$

Given that intestinal P-gp is responsible for antagonizing the permeation of harmful substances from the gut, we hypothesized that SOLE treatment may facilitate the transport of LPS through impaired efflux function of P-gp. First of all, we confirmed the effect of P-gp function on the permeability of LPS by using Caco-2 cell monolayers. As shown in Fig. 7A, the linear incline of FITC-LPS transport in the uptake direction (AP>BL) over the period of $2 \mathrm{~h}$ was significantly increased when P-gp inhibitor verapamil was used. Consistently, intracellular accumulation of FITC-LPS was increased by approximately $42 \%$ in verapamiltreated cells (Fig. 7B). TEER remained unchanged (Fig. 7C). These results demonstrated that $\mathrm{P}$-gp contributed to antagonizing the permeation of LPS in vitro.

SOLE increases FITC-LPS permeability across Caco-2 cell monolayers through the impaired P-gp function

As indicated, transport of FITC-LPS in the uptake direction (AP>BL) over the period of 2 $\mathrm{h}$ was significantly increased in cells treated with SOLE (Fig. 8A), while TEER was unchanged 
A

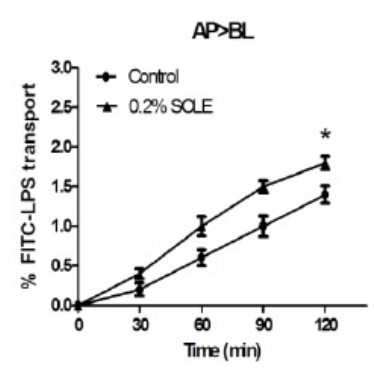

D
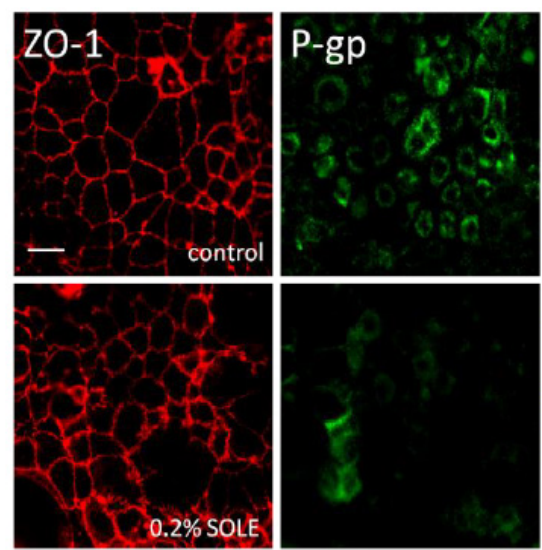

B

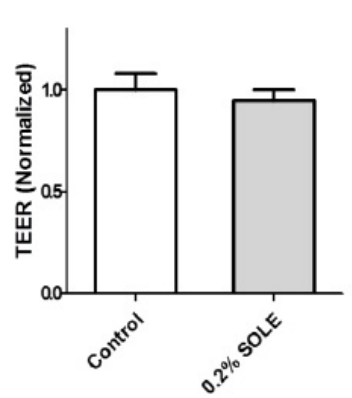

C

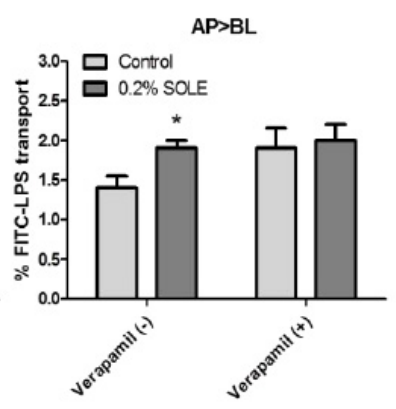

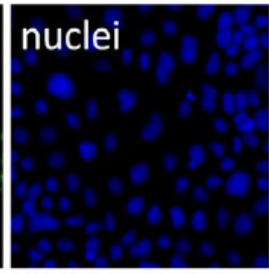
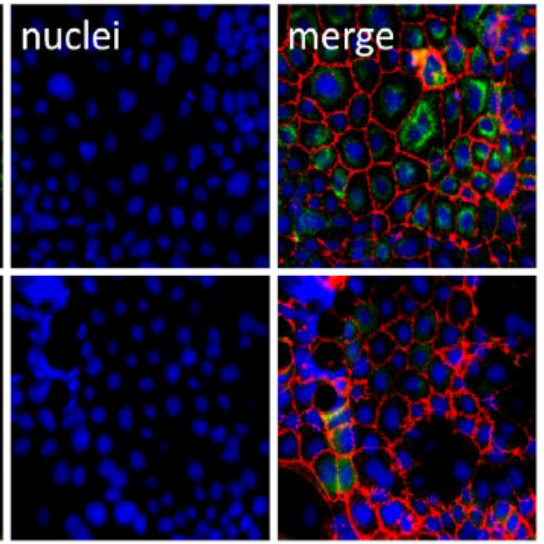

Fig. 8. SOLE increased FITC-LPS permeability through the impaired function of P-gp in vitro. (A) FITC-LPS permeability was significantly increased in the cells treated with SOLE. FITC-LPS $(50 \mu \mathrm{g} / \mathrm{ml})$ was added to apical side and the amounts of FITC-LPS in the receiver compartment were determined at 30, 60, 90, 120 min after incubation. Each point represents the means \pm SD ( $n=3$ for each group). *, $\mathrm{p}<0.05$ compared to control. (B) Integrity of monolayers was unchanged SOLE treatment. Cell integrity was evaluated by TEER following the 120 min-transport and no significant differences were observed. (C) Co-incubation with verapamil abolished the effect of SOLE on FITC-LPS transport. FITC-LPS $(50 \mu \mathrm{g} / \mathrm{ml})$ was added to apical side in the presence or absence of verapamil $(100 \mu \mathrm{M})$. The amounts of FITC-LPS in the receiver compartment were determined at 30,60, 90, $120 \mathrm{~min}$ after incubation. Each point represents the means \pm SD ( $\mathrm{n}=3$ for each group). *, p<0.05 compared to control. (D) Representative images showing Z0-1 and P-gp in cells exposed to SOLE for 48 hours. Note that treatment with SOLE resulted in an apparent loss of P-gp (green), while no significant changes in ZO-1 (red) were observed. Scale bar=75 $\mu \mathrm{m}$. Three experiments were performed that showed similar results.

(Fig. 8B) Notably, the cumulative amount of FITC-LPS transport over the period of $2 \mathrm{~h}$ was approximately increased by $23 \%$ across the cells treated with SOLE, however, co-incubation with verapamil abolished this difference between control and SOLE-treated cells (Fig. 8C). Immunofluorscence staining further demonstrated that SOLE-treated cell monolayers exhibited significantly lower immunofluorescence intensity for P-gp (green) than did the control monolayers. However, no obvious differences in the ZO-1 expression (red) were observed between the two groups (Fig. 8D), suggesting tight junction was unchanged on SOLE treatment. Taken together, these results clearly suggested that SOLE-induced increase in FITC-LPS permeability was mainly resulted from inhibition on P-gp function, other than changes in barrier function.

\section{KARGER}




\section{Discussion}

This study was designed to examine the effect of SOLE on the intestinal expression of P-gp, and its potential significance accounting for elevated permeability of LPS under TPN support. For those patients with deprivation of enteral nutrtion, TPN is the only approach of nutrition support that provides nutrients and calories intravenously. According to the United States Healthcare Cost and Utilization Project, greater than 352,000 patients received TPN in the United States in 2010 [6]. However, despite its advantages, TPN is associated with significant septic complications due in part to a loss of intestinal barrier function. While the underlying mechanisms have not been fully elucidated, a mouse model of TPN has helped identify several contributing factors. 1) Changes in the gut microbiome: a shift in intestinal microbiota from Gram-positive Firmicutes to predominantly Gram-negative Proteobacteria, which is associated with an increase in expression of proinflammatory cytokines within the mucosa, including interferon- $\gamma$ and tumor necrosis factor- $\alpha[17,18] .2)$ A concomitant loss of epithelial growth with decreased epithelial cell proliferation and increased apoptosis that in turn causes villus atrophy $[19,20] .3)$ The resulting disruption of the barrier function leads to increased intestinal permeability and bacterial translocation [21,22]. The intestinal barrier that prevents the permeation of LPS via paracellular route consists of tight junctions and adherens junctions, including occludin, claudin, tricellulin, ZO-1 and E-cadherin [23]. In TPN mice, most of these junctional proteins have been found to have a significant reduction in abundance compared to enterally fed mice [24]. However, in fact, paracellular route is not the only approach for gut microorganisms or their products to cross intestinal barrier. Mediated by membrane receptors or transporters, they may cross intestinal barrier via transcellular route as well. Thus, it can be said that if intestinal barrier-based defense system is a passive, static and structural one, then membrane transporters-based defense system would be an active, dynamic and functional one.

Given its efflux pump activity and apical localization, intestinal P-gp is considered as a natural defense mechanism that antagonizes the permeation of harmful substances from the gut via transcellular route. By using Caco- 2 cells and mdr $1 \mathrm{a}^{-/}$mice, Neudeck et al demonstrated that P-gp is involved in host defense against L. monocytogenes, suggesting that reduced P-gp on the apical surface of the enterocyte may constitute a "functional defect" in the intestinal barrier, rendering the host susceptible to bacteria [25]. Additionally, Hayashi et al showed that colonic epithelial cells have the specific transcellular transport systems for LPS, and LPS taken up into the cells can be excreted by P-gp [26]. By studying the crystal structures of mouse mdr1a, Zahida Parveen demonstrated that P-gp shares the overall architecture with two homodimeric bacterial exporters, Sav1866 and MsbA [27], providing structural evidence of a physiological role of P-gp in maintaining gut health. These results solidly indicated the protective role of intestinal P-gp in preventing luminal microorganisms and toxins from entering the systemic circulation. In fact, intestinal P-gp is related to intestinal epithelial injury under a variety of physiological and pathological conditions [2830].Therefore, whether intestinal P-gp is altered by TPN and what is the clinical significance beyond this alteration raised our great interest.

In this study, we found that SOLE, as one of the main ingredients in PN formula, directly induced down-regulation of P-gp expression and in turn facilitated the permeation of LPS via transcellular route in vitro. In Fig. 1, PN at 1\% concentration was initially used because this concentration of PN solution elicited significant inhibitory effect on P-gp function, without any detectable cytotoxicity (determined by CCK-8, data not shown) in preliminary experiment. The mechanisms underlying P-gp regulation involve at least at two signaling pathways, ERK and PI3K/Akt [31-33]. In the preliminary study, both were examined by using specific inhibitors. The results showed that inhibition of ERK significantly attenuated the regulation on P-gp after SOLE treatment, while inhibition of PI3K/Akt slightly attenuated this impact (data not shown). Considering that nuclear factor kappa B (NF-KB) is involved in P-gp regulation and serves as a common downstream target of both ERK and PI3K/Akt pathway [34-36], we initially investigated the role of NF-KB in the SOLE-induced regulation 
on P-gp. However, none of total amount of NF-KB, phosphorylation level of NF-KB, or subcellular distribution of NF-KB was changed following SOLE treatment (data not shown). Therefore, NF-KB does not appear to be involved in this system. Instead, we examined FOXO 3a, a member of FOXO subfamily belonging to forkhead family, which has been recently identified as a transcriptional enhancer of P-gp [15]. In this study, though total amount as well as phosphorylation level of FOXO 3a remained stable, subcellular distribution of p-FOXO 3a was significantly altered by SOLE treatment (Fig. 6). Notably, this SOLE-induced nuclear exclusion of FOXO 3a could be abolished by inactivation of ERK. Taken together, we propose that SOLE activates ERK pathway, which in turn phosphorylates FOXO 3a. As a result, phosphorylated FOXO 3a translocates from nucleus to cytoplasm and loses its transcriptional activity on P-gp, leading to down-regulation of P-gp and consequent increase in permeability of LPS. However, these in vitro results have not been confirmed in vivo, so it will be of great importance to carry this out in future investigations.

In summary, we demonstrated that SOLE increases intestinal permeability of LPS in vitro by down-regulation of P-gp via ERK-FOXO 3a pathway. Our findings provided direct evidence that mechanisms underlying elevated permeability of LPS under TPN support are not limited to intestinal barrier function. Impaired efflux function of P-gp that facilitates the permeability of LPS via transcellular route may be another reason.

\section{Acknowledgements}

This work was funded by Shanghai Municipal Health and Family Planning Commission (20144Y0087) and Ph.D Programs Foundation of Shanghai Jiaotong University School of Medicine (BXJ201620).

\section{Disclosure Statement}

The authors have declared no conflict of interest.

\section{References}

1 Van Gossum A, Cabre E, Hebuterne X, Jeppesen P, Krznaric Z, Messing B, Powell-Tuck J, Staun M, Nightingale J: ESPEN Guidelines on Parenteral Nutrition: Gastroenterology. Clin Nutr 2009;28:415-427.

2 Berger MM: The 2013 Arvid Wretlind lecture: Evolving concepts in parenteral nutrition. Clin Nutr 2014;33:563-570.

3 Anastasilakis CD, Ioannidis 0, Gkiomisi AI, Botsios D: Artificial nutrition and intestinal mucosal barrier functionality. Digestion 2013;88:193-208.

4 Deitch EA: Bacterial translocation of the gut flora. J Trauma 1990;30:S184-S189.

5 Balzan S, de Almeida QC, de Cleva R, Zilberstein B, Cecconello I: Bacterial translocation: Overview of mechanisms and clinical impact. J Gastroenterol Hepatol 2007;22:464-471.

6 Demehri FR, Barrett M, Ralls MW, Miyasaka EA, Feng Y, Teitelbaum DH: Intestinal epithelial cell apoptosis and loss of barrier function in the setting of altered microbiota with enteral nutrient deprivation. Front Cell Infect Microbiol 2013;3:105.

7 Feng Y, Teitelbaum DH: Tumour necrosis factor--induced loss of intestinal barrier function requires TNFR1 and TNFR2 signalling in a mouse model of total parenteral nutrition. J Physiol 2013;591:3709-3723.

8 Ho GT, Moodie FM, Satsangi J: Multidrug resistance 1 gene (P-glycoprotein 170): An important determinant in gastrointestinal disease? Gut 2003;52:759-766.

9 Feng Y, Browner P, Teitelbaum DH: Effects on varying intravenous lipid emulsions on the small bowel epithelium in a mouse model of parenteral nutrition. JPEN J Parenter Enteral Nutr 2013;37:775-786. 


\section{Cellular Physiology Cell Physiol Biochem 2016;39:1581-1594

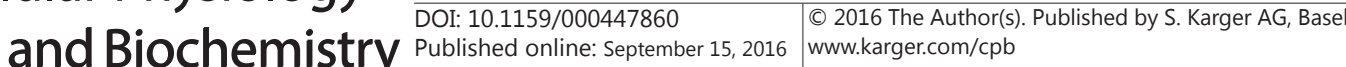 \\ Yan et al.: Soybean Oil Downregulates P-gp Expression}

10 Guzy C, Schirbel A, Paclik D, Wiedenmann B, Dignass A, Sturm A: Enteral and parenteral nutrition distinctively modulate intestinal permeability and T cell function in vitro. Eur J Nutr 2009;48:12-21.

11 Yan J, Jin G, Du L, Yang Q: Modulation of intestinal folate absorption by erythropoietin in vitro. Mol Pharm 2014;11:358-366.

12 Lee SD, Osei-Twum J, Wasan KM: Dose-Dependent targeted suppression of p-glycoprotein expression and function in caco-2 cells. Molecular Pharmaceutics 2013;10:2323-2330.

13 Drewe J, Beglinger C, Fricker G: Effect of ischemia on intestinal permeability of lipopolysaccharides. Eur J Clin Invest 2001;31:138-144.

14 Thakur S, Rahat B, More D, Kaur J: Reduced SP1-mediated transcriptional activation decreases expression of intestinal folate transporters in response to ethanol exposure. Mol Nutr Food Res 2015

15 Hui RC, Francis RE, Guest SK, Costa JR, Gomes AR, Myatt SS, Brosens JJ, Lam EW: Doxorubicin activates FOX03a to induce the expression of multidrug resistance gene ABCB1 (MDR1) in K562 leukemic cells. Mol Cancer Ther 2008;7:670-678.

16 Yang JY, Zong CS, Xia W, Yamaguchi H, Ding Q Xie X, Lang JY, Lai CC, Chang CJ, Huang WC, Huang H, Kuo HP, Lee DF, Li LY, Lien HC, Cheng X, Chang KJ, Hsiao CD, Tsai FJ, Tsai CH, Sahin AA, Muller WJ, Mills GB, Yu D, Hortobagyi GN, Hung MC: ERK promotes tumorigenesis by inhibiting FOXO3a via MDM2-mediated degradation. Nat Cell Biol 2008;10:138-148.

17 Feng Y, Teitelbaum DH: Epidermal growth factor/TNF-alpha transactivation modulates epithelial cell proliferation and apoptosis in a mouse model of parenteral nutrition. Am J Physiol Gastrointest Liver Physiol 2012;302:G236-G249.

18 Miyasaka EA, Feng Y, Poroyko V, Falkowski NR, Erb-Downward J, Gillilland MR, Mason KL, Huffnagle GB, Teitelbaum DH: Total parenteral nutrition-associated lamina propria inflammation in mice is mediated by a MyD88-dependent mechanism. J Immunol 2013;190:6607-6615.

19 Feng Y, McDunn JE, Teitelbaum DH: Decreased phospho-Akt signaling in a mouse model of total parenteral nutrition: A potential mechanism for the development of intestinal mucosal atrophy. Am J Physiol Gastrointest Liver Physiol 2010;298:G833-G841.

20 Wildhaber BE, Lynn KN, Yang H, Teitelbaum DH: Total parenteral nutrition-induced apoptosis in mouse intestinal epithelium: Regulation by the Bcl-2 protein family. Pediatr Surg Int 2002;18:570-575.

21 Feng Y, Sun X, Yang H, Teitelbaum DH: Dissociation of E-cadherin and beta-catenin in a mouse model of total parenteral nutrition: A mechanism for the loss of epithelial cell proliferation and villus atrophy. J Physiol 2009;587:641-654.

22 Kristof K, Madach K, Sandor N, Ivanyi Z, Kiraly A, Erdei A, Tulassay E, Gal J, Bajtay Z: Impact of molecular mimicry on the clinical course and outcome of sepsis syndrome. Mol Immunol 2011;49:512-517.

23 Van Itallie CM, Anderson JM: The molecular physiology of tight junction pores. Physiology (Bethesda) 2004;19:331-338.

24 Sun X, Yang H, Nose K, Nose S, Haxhija EQ, Koga H, Feng Y, Teitelbaum DH: Decline in intestinal mucosal IL10 expression and decreased intestinal barrier function in a mouse model of total parenteral nutrition. Am J Physiol Gastrointest Liver Physiol 2008;294:G139-G147.

25 Neudeck BL, Loeb JM, Faith NG, Czuprynski CJ: Intestinal P glycoprotein acts as a natural defense mechanism against Listeria monocytogenes. Infect Immun 2004;72:3849-3854.

26 Hayashi M, Tomita M: Mechanistic analysis for drug permeation through intestinal membrane. Drug Metab Pharmacokinet 2007;22:67-77.

27 Parveen Z, Stockner T, Bentele C, Pferschy S, Kraupp M, Freissmuth M, Ecker GF, Chiba P: Molecular dissection of dual pseudosymmetric solute translocation pathways in human P-glycoprotein. Mol Pharmacol 2011;79:443-452.

28 Mercado-Lubo R, McCormick BA: The interaction of gut microbes with host ABC transporters. Gut Microbes 2010;1:301-306.

29 Kugai M, Uchiyama K, Tsuji T, Yoriki H, Fukui A, Qin Y, Higashimura Y, Mizushima K, Yoshida N, Katada K, Kamada K, Handa O, Takagi T, Konishi H, Yagi N, Yoshikawa T, Shirasaka Y, Tamai I, Naito Y, Itoh Y: MDR1 is related to intestinal epithelial injury induced by acetylsalicylic acid. Cell Physiol Biochem 2013;32:942950.

30 Toklu HZ, Kabasakal L, Imeryuz N, Kan B, Celikel C, Cetinel S, Orun O, Yuksel M, Dulger GA: A study comparing the efficacy of antimicrobial agents versus enzyme (P-gp) inducers in the treatment of 2,4,6 trinitrobenzenesulfonic acid-induced colitis in rats. J Physiol Pharmacol 2013;64:439-451. 


\section{Cellular Physiology Cell Physiol Biochem 2016;39:1581-1594 \begin{tabular}{ll|l} 
DOI: 10.1159/000447860 & $\begin{array}{l}\text { O 2016 The Author(s). Published by S. Karger AG, Basel } \\
\text { www.karger.com/cpb }\end{array}$
\end{tabular}}

Yan et al.: Soybean Oil Downregulates P-gp Expression

31 Jin W, Lu Y, Li Q Wang J, Zhang H, Chang G, Lin Y, Pang T: Down-regulation of the P-glycoprotein relevant for multidrug resistance by intracellular acidification through the crosstalk of MAPK signaling pathways. Int J Biochem Cell Biol 2014;54:111-121.

32 Ma H, Cheng L, Hao K, Li Y, Song X, Zhou H, Jia L: Reversal effect of ST6GAL 1 on multidrug resistance in human leukemia by regulating the PI3K/Akt pathway and the expression of P-gp and MRP1. PLoS One 2014;9:e85113.

33 Yun M, Lee D, Park MN, Kim EO, Sohn EJ, Kwon BM, Kim SH: Cinnamaldehyde derivative (CB-PIC) sensitizes chemo-resistant cancer cells to drug-induced apoptosis via suppression of MDR1 and its upstream STAT3 and AKT signalling. Cell Physiol Biochem 2015;35:1821-1830.

34 Li Y, Huang L, Zeng X, Zhong G, Ying M, Huang M, Bi H: Down-regulation of P-gp expression and function after Mulberroside a treatment: Potential role of protein kinase $\mathrm{C}$ and NF-kappa B. Chem Biol Interact 2014;213:44-50.

35 Zhao BX, Sun YB, Wang SQ, Duan L, Huo QL, Ren F, Li GF: Grape seed procyanidin reversal of p-glycoprotein associated multi-drug resistance via down-regulation of NF-kappaB and MAPK/ERK mediated YB-1 activity in A2780/T cells. PLoS One 2013;8:e71071.

36 Liu X, Guo CY, Ma XJ, Wu CF, Zhang Y, Sun MY, Pan YT, Yin HJ: Anti-inflammatory effects of tanshinone IIA on atherosclerostic vessels of ovariectomized ApoE mice are mediated by estrogen receptor activation and through the ERK signaling pathway. Cell Physiol Biochem 2015;35:1744-1755. 